\title{
Polling: A New Randomized Sampling Technique For Computational Geometry
}

\author{
John H. Reif* and Sandeep Sen* \\ Computer Science Department \\ Duke University \\ Durham, N.C. 27706
}

\section{Abstract}

We introduce a new randomized sampling technique, called Polling which has applications to deriving efficient parallel algorithms. As an example of its use in computational geometry, we present an optimal parallel randomized algorithm for intersection of half-spaces in three dimensions. Because of well-known reductions, our methods also yield equally efficient algorithms for fundamental problems like the convex hull in three dimensions, Voronoi diagram of point sites on a plane and Euclidean minimal spanning tree. Our algorithms run in time $\mathrm{T}=\mathrm{O}(\operatorname{logn})$ for worst-case inputs and uses $\mathrm{P}=$ $O(n)$ processors in a CREW PRAM model where $\mathbf{n}$ is the input size. They are randomized in the sense that they use a total of only $\mathrm{O}\left(\log ^{2} n\right)$ random bits and terminate in the claimed time bound with probability $1-n^{-\alpha}$ for any $\alpha>0$. They are also optimal in $P \cdot T$ product since the sequential time bound for all these problems is $\Omega(n \log n)$. The best known deterministic parallel algorithms for 2-D Voronoi-diagram and 3-D Convex hull run in $O\left(\log ^{2} n\right)$ and $O\left(\log ^{2} n \log * n\right)$ time respectively while using $O(n)$ processors.

\section{Introduction}

\subsection{Background and previous work}

Designing efficient parallel algorithms for various fundamental problems in computational geometry has received much attention in the last few years. After some early work by Chow [4] in her thesis, Aggarwal et al. [1]

Research supported in part by Airforce Contract AFSOR-870386, Office of Naval Research Contract N00014-87-K-0310, National Science Foundation Contract CCR-8696134, DARPA/ARO Contract DAAL03-88-K-0185, DARPA/ISTO Contract N0001488-K-0458.

Permission to copy without fee all or part of this material is granted provided that the copies are not made or distributed for direct commercial advantage, the ACM copyright notice and the title of the publication and its date appear, and notice is given that copying is by permission of the Association for Computing Machinery. To copy otherwise, or to republish, requires a fee and/or specific permission. developed some general techniques for designing efficient parallel algorithms for fundamental geometric problems. Most of the problems tackled in that paper had $\Theta(n \log n)$ sequential complexity and the authors presented parallel algorithms which used a linear number of processors and $\operatorname{ran}$ in $\mathrm{O}\left(\log ^{k} n\right)$ time (k being typically 2,3 or 4 ) in size of the input. Consequently, a majority of the algorithms were not optimal in $P \cdot T$ bounds. A number of the problems in the original list (in [1]) have now been successfully resolved as far as $\mathrm{O}(\operatorname{logn})$ time, $\mathbf{n}$ processors algorithms are concerned, mainly due to work by Atallah, Cole and Goodrich [2]. They extended the techniques used by Cole [9] for his parallel mergesort algorithm and used a date-structure called plane-sweep tree (first proposed by Aggarwal et al. [1]) to arrive at the optimal algorithms. Perhaps the two most important problems which have been eluding such efforts are the 2-D Voronoi diagram problem and the convex hull of points in 3-space. These are very fundamental problems in computational geometry and optimal algorithms for these problems would imply corresponding optimal solutions for a multitude of other problems.

A very general definition of Voronoi diagram given by Edelsbrunner [14] is as follows:

Let $\mathrm{S}$ be a finite set of subsets of $E^{d}$ and for each $\mathrm{s} \in \mathrm{S}$ let $d_{s}$ be a mapping of $E^{d}$ to positive real numbers; we call $d_{s}(p)$ the distance function of $\mathrm{s}$. The set $\left\{p \in E^{d}: d_{s}(p)<d_{t}(p)\right.$, $\mathrm{t} \in \mathrm{S}-\{\mathrm{s}\}\}$ is the Voronoi cell of $\mathrm{s}$ and the cell complex defined by the Voronoi cells of all subsets in $\mathrm{S}$ is called the Voronoi diagram of S.

In this paper, we confine ourselves to the case where $S$ is a set of points in $E^{2}$ and the distance function is the $L_{2}$ metric. In mathematical literature, Voronoi diagrams appeared as early as in 1850 (due to Dirichlet) and again in 1907 due to Voronoi. Problems about packing and coverings of space by balls and other convex figures were among the first major applications of such diagrams. Shamos and Hoey [26] introduced Voronoi diagrams to computer science and and since then a considerable amount of research has been devoted for deriv- 
ing efficient sequential algorithms for the 2-D Voronoi diagram problem $([15,25,7])$. The Voronoi diagram is a very versatile tool for obtaining efficient solutions of some important proximity problems and is also a fundamental mathematical object in its own right. A large number of the problems can be solved in linear or $O(n \log n)$ time from the information contained in the Voronoi diagram that includes all-points nearest neighbor, Euclidean minimal spanning tree, diameter, smallest enclosing circle among others.

Since there are sequential algorithms for Voronoidiagrams that run in time $\Theta(n \log n)$, it is a fundamental question if there is a parallel algorithm that runs in $\mathrm{O}(\log n)$ time using $\mathbf{n}$ processors. Aggarwal et al. have given a $O\left(\log ^{2} n\right)$ time, $O(n)$ processors algorithm but it appears that one would require very different techniques to eliminate the $O(\log n)$ factor. Cole and Goodrich [10] reiterated the difficulties posed by this problem, when they provided some more applications of their cascadedmerging technique but were unable to extend it to the Voronoi diagram problem. In this paper we settle this question by presenting a randomized algorithm for this problem that runs in $\mathrm{O}(\operatorname{logn})$ time and uses $n$ processors in a shared memory model of parallel computation. The reader should note that the lower-bound of $\Omega(n \log n)$ also applies to the randomized algorithms by a reduction of sorting to (1-dimensional) Voronoi-diagrams. Levcopoulos, Katajainen and Lingas [20] presented an optimal expected time algorithm for Voronoi diagrams for randomly chosen set of input points; in contrast our algorithm makes no assumption about the input distribution and is optimal for the worst-case input.

Convex hulls in 3-D has a widc range of applications ranging from computer graphics to design automation to pattern recognition to operations research. Convex hulls in three dimensions can also be constructed sequentially in $\Theta(n \log n)$ time where as the best known deterministic parallel algorithm due to Dadoun and Kirkpatrick [11] runs in $\mathrm{O}\left(\log ^{2} n \log * n\right)$ time using $\mathrm{n}$ processors. In this paper we actually describe an optimal randomized parallel algorithm for constructing convex hulls in Euclidean 3-space. Due to a well-known reduction from 2-D Voronoi diagrams to 3-D convex hulls, we get an equally efficient algorithm for the first problem as an immediate corollary.

\subsection{Random-sampling and Polling in computational geometry}

Randomization has been successfully used in a wide number of applications (for example see $[16,24,27]$ ) and has recently been used to obtain efficient algorithms in computational geometry. Clarkson [5, 6, 7], Haussler and Welzl [17], and Mulmuley [21] used random sampling techniques to derive better upper-bounds for a large number of problems including the post-office problem, higher-order Voronoi diagrams, segment intersec- tions, linear programming. higher-dimensional convex hulls. The general approach taken by these algorithms as follows: a randomly chosen subset $R$ of the input set $S$ is used to partition the problem into smaller ones. Clarkson [7] proved that for a wide class of problems in computational geometry, the expected size of each subproblem is $\mathrm{O}(|S| /|R|)$ and moreover the expected total size of the subproblems is $\mathrm{O}(|S|)$. A random subset $\mathrm{R}$ which satisfies these conditions for fixed constant multiples is called a 'good' sample and is called 'bad' otherwise. Clarkson's results show that by using a straightforward random sampling technique any randomly chosen subset is good with constant probability; implying that it can also be 'bad' with constant probability. Consequently, his methods yielded expected resource bounds but cannot be used to obtain high-likelihood bounds (i.e. bounds that hold with probability $1-1 / n^{\alpha}$ for any $\alpha>0$ ). This makes it very difficult to extend his methods in the context of parallel algorithms due to the recursive nature of the algorithms. In particular the expected bounds at each recursive call are not strong enough to bound the resources used by the entire parallel algorithm due to the following reason. In a sequential algorithm, due to the linearity property of expectation (i.e. the expectation of the sum is the sum of expectations), it suffices to bound the expected time required by individual steps. The total expected time of the sequential algorithm is the sum of expected time of the individual steps. In contrast, consider the recursive parallel algorithm as a tree where a node corresponds to a procedure and the children of a node corresponds to the parallel recursive calls made by the procedure. The time required at each level of this tree is the maximum of the time required by any node of that level. There is no known method to bound the maximum of the expectations without using higher moments. The total time required by the parallel algorithm is the time when all the procedures corresponding to the leaf nodes are completed. Typically, in a parallel algorithm, the number of leaves in the corresponding process tree is at least $n^{\epsilon}(0<\epsilon<1)$. Even if we succeed in bounding the expected time for completion of a leaf-node procedure, the expected bounds are too weak to bound the maximum of the time required by all such processes.

The above problem can be dealt with by developing a technique for choosing samples that are 'good' (as defined above) with high probability. By doing so we shall show that a leaf-node process terminates in a given time bound with probability $1-1 / n^{\alpha}$ for any $\alpha>0$. In particular, for $\alpha>1$, this implies that the failure probability for the entire algorithm is less than $1 / n^{\alpha-1}$ (since there can be at most $O(n)$ leaf-level processes). We introduce a technique called polling to obtain a 'good' sample with high probability with relatively small overhead. Roughly speaking, we choose a number, $p(n)$, of random subsets (typically $\mathrm{p}(\mathrm{n})=\mathrm{O}(\log n))$ independently and determine which of these subsets is 'good' and with 
high probability one of them is 'good'. This schemc, though effective, is not very efficient since we have to repeat the procedure $p(n)$ times. Howcver, we show that we can draw conclusions about the 'goodness' of a sample very accurately by using only a fraction (typically $1 /\left(p(n)^{O(1)}\right)$ of the input which then makes the Polling scheme very efficient. This is actually very similar to the idea of polling a small fraction of the population to find out how the entire population would behave and hence the name. This turns out to be crucial in bounding the total running time of the parallel algorithm. In addition to the applications in obtaining the improved results in this paper in computational geometry, Polling appears to be a general tool for obtaining improved parallel randomized algorithms. A similar idea had been used previously by Rajasekaran and Reif [22] for their optimal integer sorting algorithm.

Note that the second property of a 'good' sample i.e. that of bounding the total size of the sub-problems is not an issue in 1-dimensional problems. In the parallel sorting algorithms of Reischuk[20] and Flashsort[19] where the total size of the subproblems always equals the input size. This is another reason why the straightforward random sampling techniques do not carry over to the recursive algorithms. Clarkson[8] circumvents this problem by limiting the number of recursive levels by a fixed constant. By using recursion over $\mathbf{s}(\mathbf{n})$ steps the problem size could grow by a multiplicative factor of $2^{\Omega(s(n))}$ if the sum of the subproblems increases by only a constant factor over the parent-problem at every recursive call. This could seriously affect the efficicncy of the algorithms, especially when we are looking for optimal algorithms. We need additional arguments to bound the total size of the sub-problems at any level of recursive calls (independent of the level number).

\subsection{Main results}

The main result in this paper can be summarized as following:

Theorem: There exists a randomized algorithm in the CREW PRAM model for constructing the intersection of $n$ half-spaces in three dimensions that runs in $O(\operatorname{logn})$ time for any input with probability $>1-1 / n^{\alpha}$ (for any given $\alpha>0$ ) using $n$ processors. Moreover, we can also limit the total number of random bits used by our algorithm to $O\left(\log ^{2} n\right)$.

The above theorem immediately implies equally efficient algorithms for the following problems from well-known reductions

\section{(i) Convex-hull of points in 3-dimensions \\ (ii) Voronoi-diagram of point sites in a plane \\ (iii) Euclidean Minimal spanning tree}

The previously best-known algorithms for all these problems are sub-optimal by at least an $O(\log n)$ factor in time complexity.
We adopt a top-down approach in describing the algorithm. In the section 2 we list some of the preliminary results that will be used as low-level procedures in the algorithm and some probabilistic notations used to aid the analysis. In section 3, we sketch a very high level description of the algorithm that uses the straight-forward random sampling (without polling) and if implemented in a straightforward manner wouldn't be very efficient. In section 4, we give a formal description of polling and its probabilistic analysis. In section 5 we describe an efficient procedure for for carrying out the divide step of the algorithm. In section 6 , we present probabilistic arguments for bounding the total time of the algorithm with high likelihood and bound the number of processors needed at any single step to complete the analysis.

\section{Some preliminary results and overview}

\subsection{Model of computation and nota- tions}

Throughout this paper we will be using the CREW PRAM model which is the synchronous shared memory model of parallel computation in which processors may simultaneously read from a memory location but are not allowed to write concurrently. At each step, a processor is allowed to perform a real-arithmetic operation consistent with standard models used for sequential geometric algorithms. Moreover, each processor has access to a random-number generator that returns in unit time a truly random number of $O(\log n)$ bits. However, see in section 6.3 , where we limit the use of truly random bits.

The term very high likelihood (probability) is used in this paper to denote probability $>1-n^{-\alpha}$ for some $\alpha>1$ where $n$ is the input size. Just like the big-O function serves to represent the complexity bounds of deterministic algorithms, we shall use Õ to represent complexity bounds of the randomized algorithms. We say that a randomized algorithm has resource bound O $(\mathrm{f}(\mathrm{n}))$ if there is a constant $\mathrm{c}$ such that the resource used by the algorithm is no more than $c \alpha f(n)$ with probability $\geq 1-1 / n^{\alpha}$ for any $\alpha>1$. (An equivalent definition will be bounding the resource by $\alpha \cdot f(n)$ with probability greater than $1-n^{-c \alpha}$ and in the rest of the paper they will be used in an interchangeable manner). Note that an algorithm whose expected resource bound is $O(f(n))$ does not have any better confidence interval beyond using Markov's inequality i.e. the probability that it exceeds the resource bound by a factor $k$ is less than $1 / k$. This implies that the failure probability does not diminish as rapidly as the high likelihood bounds. High-likelihood bounds are especially useful for parallel algorithms, where we need to bound the time complexity of all the processes. In contrast, the expected bounds 
as used by Clarkson[8] are difficult to use to bound the overall maximum time for all processes.

We will be using the term high-likelihood in a variety of situations throughout this paper that may look different from the canonical form given in the previous paragraph. We illustrate this with two lemmas which will be of use later.

Lemma 2.1 The union of $k$ events ( $k$ being any fixed integer), each of which succeeds with high probability also succeeds with high probability.

Note that if the failure probability of event $i$ is $<1 / n^{\alpha_{i}}$ the failure probability of the union of the events is less than $\sum_{i=1}^{k} n^{-\alpha_{i}}<k / n^{\alpha}$ where $\alpha=\min \left(\alpha_{1}, . ., \alpha_{k}\right)$. This is less than $n^{-(\alpha-\delta)}$ for any $\delta>0$.

Lemma 2.2 If an algorithm consists of kloglogn ( $k$ is any positive integer) nested procedure calls such that the running time of the procedure at level $i(1<i<$ $k \log \log n)$ exceeds $c \alpha \log \left(n^{1 / \sigma^{i}}\right)$, ( $\sigma$ is a constant greater than 1) with probability less than $1 / n^{\alpha / \sigma^{i}}$, then the entire program executes in time $\tilde{O}(\log n)$.

A rigorous proof of this assertion can be found in Reif and Valiant [24] (Theorem A).

For the rest of the paper, we assume that the success probability requircd by the algorithm is given, so that given $n$, we can fix $\alpha$. From this, one can compute the required probability of success at every individual step of the algorithm even though we do not provide explicit formulae. Also, for convenience of notations, functions of $n$ that may not be necessarily integral valued like loglogn or nsupe will actually denote the ceiling of such values i.e. $\lceil\log \log n\rceil$ and $\left\lceil n^{\epsilon}\right\rceil$. This does not affect the asymptotic bounds of the algorithm.

\subsection{Useful results}

In the remainder of the paper we shall assume that the half-spaces are described as inequalities of the form ax + by $+\mathrm{cz}+\mathrm{d} \geq 0$. The following observation is useful for constructing the intersection of a random subset of half-spaces that is used to split up the problem evenly.

Lemma 2.3 The intersection of a given set of $n$ halfspaces can be computed in $O(\log n)$ time using $n^{4}$ processors in a CREW PRAM model.

Proof: Assuming non-degeneracy (i.e. no 4 planes intersect at a common point), there are $O\left(n^{3}\right)$ candidate vertices for vertices of the convex hull (of the intersection). For each vertex, test whether it is a vertex of the convex hull by checking if it satisfies all the equations defining the half-spaces. This can be done trivially in $\mathrm{O}(\log n)$ time using $\mathrm{n}$ processors for each candidate point. Only the vertices would survive. Determine the faces of the convex hull by identifying planes that contain 3 vertices of the intersection.
Lemma 2.4 Given a set of $n$ half-spaces, it is possible to compute their intersection in $O\left(\log ^{3} n\right)$ time using $n$ processors in a CREW PRAM model.

Proof: Follows immediately from Aggarwal et al. [1].

This result is useful to stop the recursion at a level when the problem size is small (typically $O\left(\log ^{k} n\right)$ for some integer $\mathrm{k}$ ) and solve the problem directly. Note that any polylog-time algorithm using a linear number of processors would again suffice for our purpose. At this stage the problem size is so small that using a suboptimal algorithm will not affect the asymptotic complexity of the algorithm.

\section{A naive random sampling al- gorithm and its shortcomings}

Before we embark on a formal proof of the main theorem, let us give an informal description of the algorithm using the straight-forward random sampling strategy (as used by Clarkson [7]). We intentionally leave out Polling from this preliminary discussion to illustrate the pitfalls of using naive sampling strategies for parallel algorithms. We shall assume for the time being that we know a point $p^{*}$ in the intersection of the $\mathrm{n}$ half-spaces and later show how to determine such a point efficiently. Using a random subset of $S$, we split the original problem evenly into smaller sized problems and then apply the algorithm recursively to each of the problems. By using a random subset of size $n^{\epsilon},(0<\epsilon<1)$ we split up the problem into sub-problems of expected size $n^{1-\epsilon}$. This results in a recurrence of the form $\bar{T}(n)=\bar{T}\left(n^{1-\epsilon}\right)$ $+f(n)$, where $f(n)$ is the time for dividing the problem. If $f(n) \leq \tilde{O}(\log n)$ (which requires the use of Polling that we describe in section 4), we have an algorithm whose expected running time is bounded by $\mathrm{O}(\operatorname{logn})$. We further need to show that the number of processors required at each step of the algorithm is $O(n)$.

\section{Algorithm}

Input: A set $\mathbf{S}$ of $\mathbf{n}$ half-spaces $H_{1}, H_{2}, \ldots H_{n}$. Output: The output convex hull $\mathcal{C}$ which is intersection of the $\mathbf{n}$ half-spaces.

(1) Choose a random subset $\mathrm{R} \subset \mathrm{S}$ of half-spaces such that $|R|=n^{\epsilon}$ (for some $\epsilon, 0<\epsilon<1$ that we shall determine during the course of analysis).

2) Find the intersection of the $R$ half-spaces and again wlog assume that there is no degeneracy i.e. each vertex is the intersection of exactly three planes. Take a fixed plane and cut up each face of the polyhedron with parallel translates of this plane passing through the vertices. Thus each face is a trapezoid. Further, partition each trapezoid with a diagonal so that each face is triangular. For a face $F_{i}$ consisting of vertices $x_{i}, y_{i}, z_{i}$ consider the cone $C_{i}$ formed by $p^{*}$ as the apex and $F_{i}$ as the base. Let $C_{R}$ denote the number of cones. Note that $C_{R} \leq|R|$. 
3) For the S-R remaining; half-spaces (which are actually equations of planes) find the intersection of

the planes with the cones. Note that a plane may intersect more than one cone. The intersection of the $S$

half spaces is the union of the intersection of the half-spaces intersecting a cone (over all cones). That is, $\mathcal{C}$ is $\cup_{i=1}^{C_{R}} I_{i}$ where $I_{i}$ is the intersection of all half-spaces formed by $C_{i} \cap\left\{H^{j}\right\}$ for all $\mathbf{j}$.

4) If the number of planes intersecting the cone is more than a pre-determined threshold apply step 1-3 recursively to this set of set of half-spaces else solve the problem directly (using Lemma 2.4). end

The algorithm outlined above that uses a straightforward random-sampling in step (1) is only a skeleton of the actual algorithm and is not very efficient in its present form. One of the main problems is that in step 3 ), we could have the total size of the sub-problems exceed the size of the parent (calling) problem by a large factor at each recursive call. Note that bounding this increase at each recursive call by a constant factor is not sufficient. This would imply that after $O(\log \log n)$ levels, we can only bound the number of processors required at this stage by $\mathrm{O}\left(n \log ^{O(1)} n\right)$. This is where this algorithm differs from some other recursive parallel algorithms like randomized parallel sorting algorithms of $[24,25]$ where the total size of the subproblems is always bounded by the input size. We need more sophisticated methods for choosing random subset in step (1) to prevent this. We will show in section 4 how to solve this problem using Polling. Moreover, the procedure of detecting the intersection of the half-planes with the cones quickly is in itself a non-trivial task. For the rest of the paper, we concentrate on individual steps and provide the necessary modifications to prove the main theorem.

\section{Probabilistic lemmas}

\subsection{Need for Polling: an improved ran- dom sampling technique}

A crucial part of the analysis rests on showing that a random subset $R$ can be chosen efficiently in the first step of the algorithm that divides the the problem into almost equal sized sub-problems. In addition we have to show that the union of the sub-problems is almost equal to the complexity of the original problem at every stage of the recursive calls. The following result follows from Clarkson (Clarkson [7], Corollary 4.3) for any random $\mathrm{R} \subset \mathrm{S}$ with $|R|=r$.

Lemma 4.1 Let $X_{i}$ denote the set of planes intersecting cone $C_{i}$ (using the same terminology as in step 2 of the algorithm). Then the following conditions hold with with probability at least $1 / 2$ (i) $\sum_{i=1}^{C_{R}}\left|X_{i}\right| \leq k_{\text {total }}(n / r) \cdot E\left(C_{R}\right)$

(ii) $\max _{i} \mid X_{i}-\leq k_{\max }(n / r) \cdot \log r$

where $k_{\text {total }}$ and $k_{\max }$ are constants and $C_{R}$ is defined previously. .

Any subset of the input that satisfies the above conditions for some fixed constants is defined to be 'good' and otherwise 'bad'. A direct consequence of the lemma is that we can divide up the problem into almost equal size sub-problems, such that the increase in the original problem size can be bounded by at most a constant multiplicative factor of $k_{\max }$. Since our objective is to apply this recursively, we need a more sophisticated sampling algorithm to obtain a sample that is 'good' with high likelihood.

\subsection{An informal description of Polling}

The abstract idea is following. Since the above events would fail only with constant probability, the probability that the conditions would fail in $O(\log n)$ independent trials is less than $1 / n^{\alpha}$ for some $\alpha>0$. That is if we choose independently $p(n)=O(\log n)$ sets of samples, one of them is good with very high likelihood. However, to determine if a sample is 'good', we would have to carry out step $3, O(\log n)$ times each of which requires $\mathrm{O}(\log n)$ time (such a method is described in section 5). Instead, we try to estimate the the number of planes intersecting a cone $C_{i}$ using only a fraction of the input planes. For example, we can choose $c_{0} \cdot n / \log ^{d} n$ for some fixed integer $d>2$ and a constant $c_{0}$ (the actual value will be determined from the required success probability of the algorithm) of the input planes randomly for the jth sample, $R_{j}$. Let $X_{i}^{j}$ be the number of planes intersecting cone $C_{i}$ corresponding to sample $R_{j}, 1 \leq j \leq b \log n$ where $b$ is fixed integer greater than 0 which is determined from the success probability of the algorithm. $A_{i}^{j}$ be the number of planes intersecting $C_{i}$ out of the $\mathrm{n} / \log ^{d} n$ randomly chosen input planes for the same sample. Clearly, $A_{i}^{j}$ is a binomial random variable with parameters $\mathrm{n}=c_{0} \cdot n / \log ^{d} n$ and $\mathrm{p}=X_{i}^{j} / \mathrm{n}$ (see appendix). Assuming that $X_{i}^{j}$ is greater than $\bar{c}$ $\cdot \log ^{d+1} n$, for some constant $\bar{c}$, we will apply Chernoff bounds (see appendix) to tightly bound the estimates within a constant multiplicative factor. Since we do it only for $1 / \log ^{d} n$ of the input planes, the total number of operations for the $O(\log n)$ random subsets can be bounded by $O(n \log n)$ (as we show in the next section). Note that $X_{i}^{j}<\bar{c} \log ^{d+1} n$, is an easy case since $n^{\epsilon} \cdot \bar{c}$ $\log ^{d+1} n=o(n)$.

\subsection{Probabilistic analysis of Polling}

More formally, by invoking Chernoff bounds (see Appendix equations (1) and (2)), for any $\alpha>0(\alpha$ is a function of $c_{0}$ ), there exists a $c_{1}$, independent of $\mathbf{n}$, 
$\operatorname{Prob}\left(A_{i}^{j} \leq \alpha c_{1} X_{i}^{j} / \log ^{d} n\right) \leq 1 / n^{\alpha}$ and $\operatorname{Prob}\left(A_{i}^{j} \geq\right.$ $\left.c_{2} \alpha c_{0} \cdot X_{i}^{j} / \log ^{d} n\right)<1 / n^{c_{0} \alpha}<1 / n^{\alpha}\left(\right.$ for $\left.c_{0}>1\right)$. From the last two inequalities, $X_{i}^{j}$ is bounded by $L^{j}=$ $A_{i}^{j} \log ^{d} n / c_{0} c_{2} \alpha$ from below, and $U^{j}=$ by $A_{i}^{j} \log ^{d} n / c_{1} \alpha$ from above. With appropriate changes in the constants, this condition holds with high likelihood (as defined in section 2.1) for all $X_{i}^{j}$ simultaneously. We do the procedure (described in the next section) simultaneously for all the samples $R_{j}$ and choose the sample $R_{j_{0}}$ using the following simple test:

\section{Algorithm Polling}

(Let $N^{j}=\sum A_{i}^{j}$ and the let actual number of intersections be denoted by $T^{j}$ and the upper and lower bounds obtained from $N^{j}$ by $U^{j}$ and $L^{j}$ respectively).

If $k_{\text {total }} n>U^{j}$ then accept sample $R^{j}$ (since
$k_{\text {total }} n U^{j} \geq T^{j}$ ), else if $k_{\text {total }} n \leq$
$L^{j}$ then the sample is 'bad' (since $k_{\text {total }} n \leq$
$L^{j} \leq T^{j}$ ), else if $L^{j} \leq k_{\text {total }} n \leq U^{j}$, then accept the sample $R^{j_{0}}$ for which $E^{j_{0}}$ is minimum. Since both $k_{\text {total }} n$ and $T^{j \circ}$ lie in this interval this guarantees that $T^{j_{0}} \leq c_{3}$. $k_{\text {total }} n$ where $c_{3}=U^{j} / L^{j}$ which is a constant.

Recall, that from our earlier discussion at least one of the samples would satisfy conditions 1 or 3 with very high likelihood. We summarize as following :

Lemma 4.2 (Polling lemma) If we can choose a set of random splitters that expects to be 'good' (i.e. satisfies certain properties), then by using the polling algorithm, as described earlier we obtain a sample that is 'good' with high probability.

The above procedure can actually be used in a more general situation where we need 'good' samples with very high likelihood from samples that only expect to be 'good'. Moreover, according to our previous discussion, the extra amount of overhead does not affect the asymptotic work done by the algorithm, because it uses only a fraction of the input to test the samples.

\section{$5 \quad$ Finding intersections quickly}

\subsection{A locus-based approach for finding intersections}

We now focus on describing a procedure to find the intersection of planes with each of the cones, $C_{i}$. Notice that a plane may intersect more than one cone which rules out detecting the intersections sequentially. That is, if a plane intersects $n^{\delta}$ cones $(\delta>0)$, we cannot afford to detect them one after the other since we are looking for an $O(\log n)$ time procedure. Note that in the sequential case, Clarkson and Shor's [8] randomized incremental constructions give optimal expected time bounds that cannot be applied in our case.

We shall use a locus-based approach to solve this problem. This approach involves considering each query as a higher-dimensional point and partitioning the underlying space into regions providing the same answer. Thus any query problem can be reduced to a point location problem given sufficient preprocessing time and space. In our case, we have to pre-process the convex hull of the sampled half-spaces in such a way that given any plane, we should be able to report the cones that it intersects in $\mathrm{O}(\log n)$ time using at most $\mathrm{k}$ processors where $\mathrm{k}$ is the number of intersections. We shall show that the preprocessing for a convex hull of $O(n)$ size can be done in $O(\log n)$ parallel time using $O\left(n^{c}\right)$ processors, where $c$ is a fixed constant. Thus we can choose any sample of size less than $n^{1 / c}$ since we have $n$ processors.

Given a convex polyhedron in 3-D of size $O(n)$ along with an internal point which is the apex of the cones, there can exist only a polynomial (in n) number of combinatorially distinct possibilities of the way any given plane can intersect the cones. This can be seen from the following simple argument. Given any plane that intersects the polyhedron, we can perturb the plane without changing the cones it intersects so long as it remains within a fixed set of bounding vertices. Figure 1 illustrates the situation for a two-dimensional case. If we consider an equivalence relation where two lines are equivalent iff they intersect the same sets of cones then the equivalence classes correspond to the cells in the arrangement $\mathcal{A}(H)$ where $\mathbf{H}=\{\mathcal{D}(p)$ : $\mathrm{p}$ is a vertex of the convex hull or internal point and $\mathcal{D}$ is a dual transform \} (see [14] for more details). Given any query line $l$, the cones that it intersects is defined by the partition of $\mathcal{A}(H)$ that $\mathcal{D}(l)$ belongs to. This observation can be extended to hold for any dimension; in our case three. If we consider the partitions of the three-space induced by the intersections of the constraining half-spaces, these are equivalent classes with respect to the cones they intersect. Notice that even if this partitioning may not be minimal but it suffices for our purpose. All that remains to be done is pre-compute for each of these regions the cones that the corresponding planes would intersect so that for any query plane in the same equivalence class we can list off the intersecting planes by a table look-up.

\subsection{A point-location algorithm}

For the point-location problem, we use a pre-processing scheme due to Dobkin and Lipton [13] because of the ease in parallelization. The following is a fairly straightforward extension of their method

Lemma 5.1 For any set of $m$ planes in $E^{3}$, it is possible to pre-process them in $O(\operatorname{logm})$ time using $\mathcal{O}\left(\mathrm{m}^{7}\right)$ 
pracessors, such that point-location for an arbitrary query point can be done in $O(\operatorname{logm})$ time. The space required is $O\left(m^{7}\right)$.

Proof: Find the pairwise intersections of the given set of planes (there are $\mathrm{O}\left(\mathrm{m}^{2}\right)$ of them). Project the resulting lines on a plane which is not normal to any of the lines. Find the pairwise intersections of the straight lines and consider their projection on the $\mathrm{x}$-axis. There are $\mathrm{O}\left(\mathrm{m}^{4}\right)$ intervals induced by these. For each of these intervals, order the straight-lines by sorting. This can be done in $\mathrm{O}(\operatorname{logm})$ time using $\mathrm{O}\left(\mathrm{m}^{2}\right)$ processors for each of the $O\left(m^{4}\right)$ intervals. There are now $O\left(m^{6}\right)$ trapezoidal regions. For each of these, order the planes for binary-search by sorting which are totally ordered in these subdivisions. This can be done in $\mathrm{O}(\log m)$ time using $\mathrm{O}\left(\mathrm{m}^{7}\right)$ processors. The subdivisions induced by this pre-processing are homeomorphic to a 3-cube, so that given any query point it can be located in such a subdivision with 3 binary searches.

For each of the subdivisions in 3-space, we can precompute the cones that the corresponding plane intersects using $\mathrm{O}\left(n^{8}\right)$ processors. Note that these subdivisions are finer than the minimal equivalence classes i.e. more than one subdivisions could have the same set of intersecting cones. We also store the number of intersecting cones for each of the subdivisions so that while listing the number of cones each query plane intersects we can do the processor allocation easily in $O(\log n)$ time using a prefix computation. By choosing less than $n^{1 / 8}$ samples, we can complete the entire preprocessing in the required time and processor bounds.

We summarize our conclusion in this section as follows

Lemma 5.2 Step three of the algorithm uses $O(n \operatorname{logn})$ space and terminates in $O(\log n)$ time using $n$ processors in a CREW PRAM model.

Note that in the course of the entire algorithm, the concurrent reads are utilized only during the binary searches.

\section{Putting things together}

\subsection{Controlling the size of subproblems}

Even though we have shown that most of the algorithm works out as desired, there is more that needs to be covered to complete the analysis. From lemma 4, we know that the size of the problem can increase by a constant factor at each level and we wish to avoid this happen over $O(\log \log n)$ levels, which would increase the number of processors required by a polylog factor.

For this we need to quickly identify the redundant planes that do not contribute to the output complexity and eliminate them from further recursive calls. This enables us to get a global bound on the total size of the subproblems at any stage which we shall show to be linear in the input plus the output size. More specifically, we allocate the processors recursively to the cones such that the number of processors is proportional to the number of output vertices in that cone, thereby bounding the number of processors to be $O(n)$. The details of the procedure is described below. After we have found

the planes intersecting a particular cone, we categorize thern into the following types:

(a) The planes that are completely occluded by another plane in the cone and hence these cannot be a part of the output in the cone (b) Planes that are occluded because of more than one other plane in the cone i.e. there is no one plane that completely occludes them.

(c) Planes that contribute to an edge without an end-point i.e. the end-points lie in some other cones.

(d) Planes that do contribute to a vertex in the cone

To eliminate planes of type (a), we use a variant of the 3-D maxima algorithm. The $3-D$ maxima problem is defined as:

Given a set $\mathbf{S}$ of $\mathbf{n}$ points in a three-dimensional space, determine all points $p$ in $S$ such that no other point of $\mathrm{S}$ has $\mathrm{x}, \mathrm{y}$ and $\mathrm{z}$ coordinates that simultaneously exceed the corresponding coordinates of $p$.

Since cones have a triangular base there are 3 edges that join it to the apex $p^{*}$. We sort the intersections of the planes with an edge in increasing distances from the apex. We repeat this for all the three edges. Call these three edges $X, Y, Z$ and denote the intersection of a plane $h_{i}$ as $X_{i}, Y_{i}, Z_{i}$ and the ranks in the sorted list as $\mathrm{r}\left(X_{i}\right), \mathrm{r}\left(Y_{i}\right)$ and $\mathrm{r}\left(Z_{i}\right)$.

Observation 1: If a plane $A$ is occluded completely by another plane $B$ iff it is dominated on its ranks of intersection on all the three edges by plane $B$.

This gives us an effective strategy for eliminating planes of type (a) by identifying the complement of the set of the maximal elements, where we use the ranks of the intersection on the three edges as the order relation. Using the $O(\log n)$ time $n$ processors algorithm of [2], we can do this in $O(\log n)$ time.

To identify planes of type (b) (c) and (d) we construct the intersection of the 3-D convex hull $\mathcal{C}$ with each of the three faces of the cone. These are intersections of the faces with $\mathcal{C}$ that are 2-D convex hulls. These will be referred to as contours for the following discussion. The contours can be computed in $O(\log n)$ time with $n$ processors using any of the optimal 2-D convex hull algorithms. Note that these convex contours on the three faces are a part of the output and any plane that appears on this contour is a part of the final output. Consequently, a plane of type (b) cannot be a part of this 
contour. Unfortunately, there can be planes that are part of the output but are not part of any contour (consider a plane that chops off a cap of the hull within the cone). For the time being let us focus on only those planes that show up in the contours and consider the 3-D convex hull formed only by these planes within a cone. We shall refer to such a 3-D hull as a skeletal-hull. We now make following observation

Observation 2: Any plane that is is not a part of the contour on any face can intersect at most one skeletal hull.

This follows from convexity. Notice that such planes are not necessarily a part of the output but we are not aiming for an output sensitive algorithm. The previous observation guarantees that if a plane is not a part of $\mathcal{C}$ it will not survive in more that one cone when the algorithm is called recursively in the cones. The planes that do not intersect the skeletal-hull cannot be a part of $\mathcal{C}$ within the cone. See Figure 2 for a two-dimensional illustration of these cases.

A plane that contributes to only an edge of the convex hull will be a part of the contour and on exactly two faces (intersection of the edge with the cone) and hence can be identified quickly using sorting.

The above method for throwing out redundant planes ensures that going into any recursive call, the sum of the subproblems is less than $n+3 m$ where $n$ and $m$ are respectively the input and output sizes and assuming that there are no singularities (a vertex is the intersection of exactly 3 planes).

We shall now describe a procedure to construct the skeletal-hull within a cone and preprocess the skeletalhull such that queries of the kind plane-polyhedra intersection detection can be answered quickly. The latter part can be done efficiently using a hierarchical polyhedra decomposition scheme due to Dobkin and Kirkpatrick [12]. The construction of the hierarchical representation can be done in $\tilde{O}(\log n)$ time using an algorithm of Reif and Sen [23] (also discovered independently by Dadoun and Kirkpatrick [11] but the analysis given in their paper is not sufficient for our purposes). Given this representation, the plane-polyhedra intersection detection query can be answered in $O(\operatorname{logn})$ sequential time (Kirkpatrick [19]).

We shall now discuss how to construct the skeletalhulls quickly. Although the skeletal-hulls are themselves 3-D convex hulls they have a much simplcr structure. More specifically, they have the following property: all faces are unbounded (i.e. they are part of the contours. This implies that, if we construct them recursively using the same algorithm, we do not have to worry about case (b) since all planes that are part of the output will show up in the contours and this holds for any level of the recursive call. From the analysis given in the next sub-section the skeletal-hulls can be constructed in $\tilde{O}(\log n)$ time using a linear number of processors. The reader should convince himself that there is no circular- ity of arguments here. One way to look at the problem is the following : assuming that case (b) doesn't arise (i.e. all planes that are part of the output show up in the contours), the algorithm terminates in $\tilde{O}(\log n)$ time using a linear number of processors. So after having constructed the skeletal-hull for the cone, the redundant planes are quickly eliminated using the procedure outlined in the previous paragraph. Subsequently, the algorithm is called recursively on the cone - this time to build the actual hull (in contrast to the skeletal-hull).

\subsection{Final analysis}

Consider the algorithm as a tree where each node corresponds to a procedure and the children of a node representing processes corresponding to the recursive calls made by the procedure. Then the running time of the algorithm corresponds to a worst-case sequence of nested procedure calls along any path in this tree from the root to a leaf node. This process tree corresponding to the algorithm has the following property. A process at level $\mathrm{i}(1<i<\mathrm{O}(\log \log n))$ has size $\mathrm{O}\left(n^{(10 / 9)^{-i}}\right)$ and the process terminates in time $\mathrm{O}(\log$ $\left.n^{(9 / 10)^{i}}\right)\left(=(9 / 10)^{i} \mathrm{O}(\log n)\right)$ with probability greater than $1-1 / n^{(9 / 10)^{i}}$. From Lemma 2.2, any nested sequence of recursive calls exceeds time $c \gamma \log n$ with probability less than $1 / n^{\gamma}$ for any $\gamma>1$. From Lemma 2.1 in section 2.1 , it follows that all the leaf processes and hence the algorithm are completed within the same time with high likelihood. The space used is $O(n)$ at step 3 of each recursive level giving a total bound of $O(n \log \log n)$ for all the $O(\log \log n)$ recursive levels of the algorithm. This proves the main result of the paper.

Corollary 6.1 The following problems can be solved in Ó(logn) time using $n$ processors in a CREW PRAM

(i) Convex hull of a set of points in 3-D

(ii) Voronoi diagram of point-sites in plane

(iii) All-points nearest neighbor

(iv) Euclidean minimal spanning tree

Proof: (i) follows immediately because of well-known reduction of convex hulls to intersection of half-spaces. To determine an internal point $p^{*}$ in the intersection, we can determine an internal point of the convex hull and use it as the origin for the duality transform. The origin is known to be contained in the intersection of the half-spaces.

For (ii), given a set of $\mathbf{n}$ points in the plane we apply an inversive transformation given by Brown [3] to the input points to transform the problem into finding the convex hull of $\mathrm{n}$ points in 3-space.

(iii) can be obtained in $O(\log n)$ time from the Voronoidiagram.

(iv) can be obtained by running a minimal-spanning tree 
algorithm on the edges of Delaunay triangulation which is the dual graph of the Voronoi diagram.

\subsection{Bounding the number of random bits}

Since randomness has been recognized as a scarce resource, it is important to bound the amount of random bits used by our algorithm.

Lemma 6.1 The algorithm can be made to run in the same time bound using at most $O\left(\log ^{2} n\right)$ truly random bits.

Proof sketch: We use techniques of Karloff and Raghavan [18] to limit the use of truly random bits in Reischuk's parallel sorting algorithm. They use pseudorandom number sequences of the form $a+b \cdot i \bmod P, i$ $=1,2,3$. where $a_{2} b$ are truly random seeds of $O(\operatorname{logn})$ bits chosen uniformly from $Z_{P}$ for suitable primes $P$ of size $n^{O(1)}$. The main ideas are - (1) (Asymptotically) similar probabilistic bounds can be derived from Chebychev's inequality which uses only 2-way independence and (2) that the same random bits can be reused during the the different levels of recursive calls. The process tree (as defined in our previous discussion) is partitioned into groups of contiguous levels which they call bands, where each band uses $O(\log n)$ truly random bits. They show that the same random bits can be shared by all bands in a path and by all paths in the tree. The algorithm still runs in õ (logn) time. In our case, we use $\mathrm{K}$-way independence where $\mathrm{K}$ is a predetermined constant and a generalized Chebychev inequality of the form $\operatorname{Prob}\{|X| \geq t\} \leq \frac{M}{\phi(t)}$ where $M=E[\phi(t)]$. From Lemma A.1 in the appendix, bounds similar to Lemma 4.1 can be rederived using K-way independence from the generalized Chebychev's inequality. The bounds for polling can also be derived similarly from Corollary A.1. We need an extra $O(\log n)$ multiplicative factor of truly random bits for implementing Polling for which we need $\mathrm{O}(\log n)$ independently chosen random seeds of $\mathrm{O}(\log n)$ bits each. The overall effect of using these schemes is that the constants associated with the resource bounds grow larger.

\section{Concluding Remarks}

The randomized algorithms presented here the reinforces the optimism expressed in an earlier paper (Reif and Sen [23]) where we introduced randomization as an effective tool for developing parallel algorithms in computational geometry. Clarkson had demonstrated the usefulness of randomization for deriving improved expected time bounds for a large number of sequential algorithms. Although we draw from Clarkson's work, our results should be of independent interest because of many unique additional difficulties presented by the parallel environment and the techniques one needs to tackle them.

This paper describes the first $O(\log (n)$ parallel time algorithm with optimal speed-up for 3-D convex hulls and related problems, however a number of questions are left unanswered. The most obvious problem is that of designing a deterministic algorithm with same bounds. It is possible that an optimal algorithm for 2-D Voronoi-diagrams may be easier to obtain than a similar algorithm for 3-D convex-hulls. Moreover, we use the CREW PRAM model for our algorithm that raises the question if the algorithm can be made to run without the feature of concurrent reads. A more theoretical issue is that of designing sub-logarithmic time parallel algorithms for all these problems with optimal speed-ups.

An extremely important area of investigation in the field of parallel algorithms for computational geometry is development of efficient algorithms for fixed interconnection networks like hypercubes and butterfly networks. In spite of some elegant work done in the PRAM model, the currently best known results for almost all these fundamental problems except 2-D convex hulls remain sub-optimal. It appears very unlikely that the optimal algorithms would be deterministic since there are no known optimal deterministic sorting algorithms for these networks. This should encourage more research in the area of developing more sophisticated probabilistic methods for parallel computational geometry.

\section{References}

[1] A. Aggarwal, B. Chazelle, L. Guibas, C. O'Dunlaing, and C. Yap. Parallel computational geometry. Proc. of 25th Annual Symposium on Foundations of Computer Science, pages $468-477,1985$. also appears in full version in Algorithmica, Vol. 3, No. 3, 1988, pp. 293-327.

[2] M.J. Atallah, R. Cole, and M.T. Goodrich. Cascading divide-and-conquer: A technique for designing parallel algorithms. Proc. of the 28th Annual Symposium on the Foundations of Computer Science, pages 151 - 160, 1987.

[3] K.Q. Brown. Voronoi diagram from convex hulls. Informat. Process Lell., 9:223 - 228.

[4] Anita Chow. Parallel Algorithms for Geometric Problems. PhD thesis, University of Illinois at Urbana-Champaign, 1980.

[5] K.L. Clarkson. A probabilistic algorithm for the post-office problem. Proc of the 17th Annual SIGACT Symposium, pages $174-184,1985$. 
[6] K.L. Clarkson. New applications of random sampling in computational geometry. Discrete and Computational Geometry, pages 195 - 222, 1987.

[7] K.L. Clarkson. Applications of random sampling in computational geometry ii. Proc of the 4th Annual ACM Symp on Computational Geometry, pages 1 $-11,1988$.

[8] K.L. Clarkson and P. Shor. Algorithms for diametral pairs and convex hulls that are optimal, randomized and incremental. Proc. of the 4 th $A C M$ Symp. on Computational Geometry, 1988.

[9] R. Cole. Parallel merge sort. Proc. of the 27th Annual IEEE Symp. on Foundations of Computer Science, pages $511-516,1986$.

[10] R. Cole and M.T. Goodrich. Optimal parallel algorithms for polygon and point-set problems. Proc. of the 4th ACM Symp. on Computational Geometry, pages $201-210,1988$.

[11] N. Dadoun and D.G. Kirkpatrick. Parallel processing for efficient subdivision search. Proc. of the $9 r d$ Annual ACM Symp on Comput. Geom, pages 205 - 214, 1987.

[12] D. Dobkin and D. Kirkpatrick. A linear time algorithm for determining the separation of covex polyhedra. Journal of Algorithms, 6(3):381 - 392, 1985.

[13] D. Dobkin and R.J. Lipton. Multidimensional searching problems. SIAM J. on Computing, 5:181 $-186,1976$.

[14] H. Edelsbrunner. Algorithms in combinatorial geometry. EATCS Monographs on Theoetical Computer Science. Springer Verlag, 1987.

[15] S. Fortune. A sweepline algorthm for voronoi diagrams. Proc of the 2nd ACM Symp. on Comput., pages $511-516,1986$.

[16] H. Gazit. An optimal randomized parallel algorithm for finding connected components in a graph. Proc. of the 27th Annual IEEE Symp. on Foundations of Computer Science, pages $492-501,1986$.

[17] D. Haussler and E. Welzl. $\epsilon$-nets and simplex range queries. Discrete and Computational Geometry, 2(2):127 - 152, 1987.

[18] H. Karloff and P. Raghavan. Randomized algorithms and pseudorandom numbers. Proc. of the 20th Annual STOC, 1988.

[19] D.G. Kirkpatrick. private communication.

[20] C. Levcopoulos, J. Katajainen, and A. Lingas. An optimal expected-time parallel algorithm for voronoi diagrams. Scandenavian conference on theoretical computer science, 1988.
[21] K. Mulmuley. A fast planar partition algorithm 1. Proc. of the 29th IEEE FOCS, pages 580-589, 1988.

[22] S. Rajasekaran and J. Reif. Optimal and sublogarithmic time radomized parallel sorting algorithms. Technical Rept, Aiken Computing lab, Harvard University, 1986. To appear in SIAM Journal on Computing.

[23] J.H. Reif and S. Sen. Optimal randomized parallel algorithms for computational geometry. Proc. of the 16th International conference on Parallel Processing, 1987. A revised version is available as Duke University technical report CS-88-01.

[24] J.H. Reif and L.G. Valiant. A logarithmic time sort for linear size networks. Journal of the ACM, 34:60 $-76,1987$.

[25] R. Reischuk. A fast probabilistic parallel sorting algorithm. Proc. of the 22nd IEEE FOCS, pages 212 - 219, 1981.

[26] M. Shamos and D. Hoey. Closest-point problems. Proc. of the 7th ACM STOC, pages 224-233.

[27] L.G. Valiant. A scheme for fast parallel communication. SIAM J. on Computing, 11:350-361, 1982.

\section{A Appendix}

We say a random variable $\mathrm{X}$ upper-bounds another random variable $Y$ (equivalently $Y$ lower bounds $X$ ) if for all $\mathrm{x}$ such that $0 \leq x \leq 1, \operatorname{Prob}(\mathrm{X} \leq x) \leq \operatorname{Prob}(\mathrm{Y} \leq$ $\mathrm{x})$.

A Bernoulli trial is an experiment with two possible outcomes viz. success and failure. The probability of success is $p$.

A binomial variable $X$ with parameters $(n, p)$ is the number of successes in $n$ independent Bernoulli trials, the probability of success in each trial being $p$. The probability mass function of $\mathrm{X}$ can be easily seen to be

$$
\begin{aligned}
& \operatorname{Prob}(\mathrm{X} \leq \mathrm{x})= \\
& \sum_{k=0}^{x}\left(\begin{array}{c}
n \\
k
\end{array}\right) p^{n}(1-p)^{n-k}
\end{aligned}
$$

The tail end of the Binomial distribution can be bounded by Chernoff bounds. In particular the following approximations due to Angluin and Valiant are frequently used:

$$
\begin{aligned}
& \operatorname{Prob}(\mathrm{X} \geq \mathrm{m}) \leq\left(\frac{n p}{m}\right)^{m} e^{m-n p}(1) \\
& \operatorname{Prob}(\mathrm{X} \leq \mathrm{m}) \leq\left(\frac{n p}{m}\right)^{m} e^{-n p+m}(2) \\
& \operatorname{Prob}(\mathrm{X} \leq(1-\epsilon) p n) \leq \exp \left(-\epsilon^{2} n p / 2\right) \\
& \operatorname{Prob}(\mathrm{X} \geq(1+\epsilon) n p) \leq \exp \left(-\epsilon^{2} n p / 3\right)
\end{aligned}
$$


for all $0<\epsilon<1$. The last two bounds actually follow from the Chernoff bounds which (for a discrete distribution) can be stated as

$\operatorname{Prob}[\mathrm{A} \geq \mathrm{x}] \leq z^{-x} G_{A}(z)$ where $G_{A}(z)$ is the probability generating function.

To minimize the bound we substitute $\mathrm{z}=z_{0}$ that minimizes the right side expression.

Similar bounds can be derived even if $\mathrm{X}$ is not strictly a sum of independent Bernoulli variables but instead consists of a sum of variables which are only $2 \mathrm{k}$-way independent i.e. any subset of $2 \mathrm{k}$ (or less) variables are independent.

Lemma A.1 Let $X$ be a sum of $n$ gk-way independent and identical Bernoulli random variables $X_{i}, 1 \leq i \leq n$ each of which has a success probability $p$. Then for a fixed $k$ (chosen independently of $n$ ), Prob $\{|X-\mu| \geq \mu\}$ $\leq O\left(\frac{1}{\mu^{k}}\right)$ where $\mu=n p$ and $p \leq O\left(n^{-\beta}\right)$ for some $0<$ $\beta<1$.

Proof: Consider the generalized Chebychev's inequality $\operatorname{Prob}\{|X| \geq t\} \leq \frac{E(\phi(X))}{\phi(t)}$. Using $\phi(t)=t^{2 k}$ and substituting $X-E[X]$ for $X$, we get

$\operatorname{Prob}\{|X-E[X]| \geq E[X]\} \leq \frac{E\left[(X-E[X])^{2 k}\right.}{E^{2 k}[X]}$

Let us focus on the numerator - we shall show that it is $O\left(\mu^{k}\right)$ and the lemma follows. Since $\mathrm{E}[\mathrm{X}]=\sum_{i=1}^{n} E\left[X_{i}\right]$, we can write $(X-E[X])^{2 k}$ as $\left(\sum_{i=1}^{n} X_{i}-E\left[X_{i}\right]\right)^{2 k}$

In the multinomial expansion, all the terms containing $X_{i}-E\left[X_{i}\right]$ (for any i) as a factor vanish because of the $2 \mathrm{k}$-way independence property.

Note that $E\left[\prod_{s=j_{1}}^{j_{2 k}} \phi_{s}\left(X_{s}\right)\right]=\prod_{s=j_{1}}^{j_{2 k}} E\left[\phi_{s}\left(X_{s}\right)\right]$.

There are $\left(\begin{array}{c}n \\ c\end{array}\right) \cdot\left(\begin{array}{c}2 k-1 \\ c-1\end{array}\right)$ terms which have $c$ distinct non-unit product terms of the form $\left(X_{j}-E\left[X_{j}\right]\right)^{i}$ such that $i>0$ and $\sum i=2 k$. Also note that $E\left[\left(X_{j}-E\left[X_{j}\right]\right)^{i}\right]=(1-p)(-p)^{i}+p(1-p)^{i}$

We can factor out $p^{i}$ so that we can write the coefficient of $n^{c}$ as $p^{2 k} \cdot \mathrm{f}(\mathrm{p}, \mathrm{c}, \mathrm{k})$, where $\mathrm{f}$ is a function independent of $n$ and can be absorbed in the big-O notation. From our observation about the first-order terms (which vanish), the maximum value of $\mathrm{c}$ is $\mathrm{k}$. Moreover from our assumption about $p \leq O\left(n^{-\beta}\right)$, the numerator can be bound by the asymptotically dominating term $\mathrm{O}\left(n^{k} \cdot p^{k}\right)$ $=\mathrm{O}\left(\mu^{k}\right)$. Since the denominator is $\mu^{2 k}$, the lemma follows.

Corollary A.1 Prob $\{|X-\mu| \geq a \cdot \mu\} \leq O\left(\frac{1}{a^{k} \cdot \mu^{k}}\right)$ where $a$ is a constant between 0 and 1 .

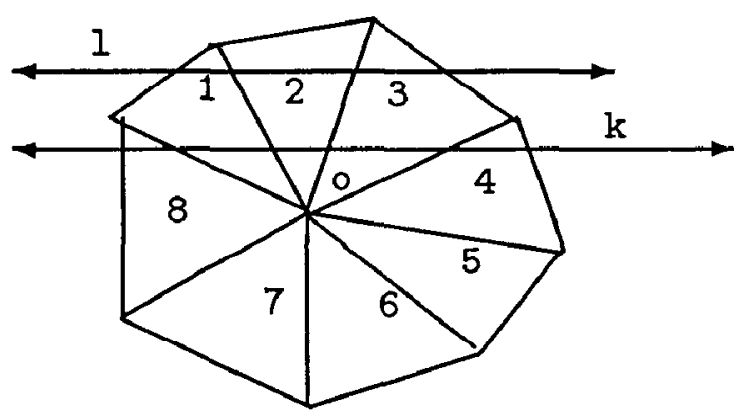

Figure 1: Lines 1 and $k$ partition the vertices differently and lie in different regions of the dual space arrangements of vertices.

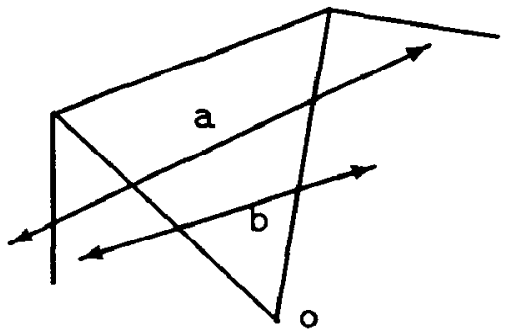

(a)

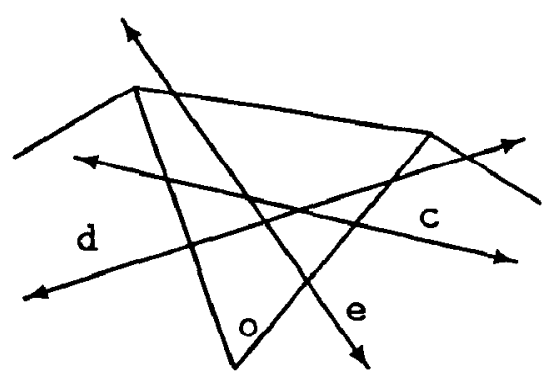

(b)

Figure 2:

(a) illustrates case (a). Line a is completely dominated by line b. (b) illustrates case (b). Line $c$ is not dominated by $d$ or e alone but by their combination. Clearly line $c$ will be eliminated from all other cones by case (a). 